\title{
Studies on Pore Characteristics of Microporous Carbons Prepared with Different Types of Silica Templates
}

\author{
S. Manocha^ and Narendra Movaliya \\ Department of Materials Science, Sardar Patel University, Vallabh Vidyanagar-388 120 Gujarat, India \\ ^e-mail: sm_manocha@rediffmail.com \\ (Received February 24, 2007; Accepted March 8, 2007)
}

\begin{abstract}
Microporous carbons with narrow pore size distribution have been successfully synthesized by using hydrolyzed and calcined silica as templates and phenol formaldehyde (pf) resin as carbon precursor. Phenol formaldehyde-silica micro composites were prepared by solution route. Subsesequently, silica templates were removed by HF leaching. Resulting carbons were steam activated. The porous carbons were characterized by nitrogen adsorption-desorption isotherm, SEM, FTIR analysis, iodine adsorption, thermogravimetry analysis, etc. Adsorption isotherms show that the porous carbon prepared from calcined silica as templates are microporous with $88 \%$ pores of size $<2 \mathrm{~nm}$ porosity and are of type I isotherm, while porous carbon prepared by using hydrolyzed silica are microporous with $89 \%$ microporosity, shows hysteresis loop at high relative pressure indicating the presence of some mesoporosity in samples. The microporosity in porous carbon materials has a bearing on the nature of silica templates used for pore formation.
\end{abstract}

Keywords : Microporous carbon, Template method, Silica template, Adsorption, SEM

\section{Introduction}

The adsorption capacity and interfacial properties of activated carbon depend on the pore structure, pore size distribution and on the chemical nature of its surface, especially the surface functional groups [1]. The adsorption in micropores and voids proceeds by volume filling, while pore size distribution influence the adsorption kinetic of gases and vapors on activated carbon. For many years, porous carbon materials have been produced by heat treatment of precursors such as coal, bitumen or biomaterials such as wood, nut shell, etc. [2,3] followed by activation process (physical or chemical method). Whilst, these can yield carbons with high surface area and with good adsorption properties, these suffer from two drawbacks as well. Firstly, owing to the disorder and inhomogeneous nature of the starting raw materials, the resulting carbon has a wide and poorly controlled pores and pore size distribution. Secondly, these carbons contain varieties of impurities, e.g. sulphur, metals, etc, depending on the characteristic of the starting precursor.

The performance of an adsorbent is determined by its surface area, pore size and surface properties, the last two of these are poorly characterized and are extremely difficult to control in commercial carbons. Controlled pore size and pore size distribution with specific surface characteristics are necessary for specific end use because the performance of porous carbon depends strongly on pore structure as well as surface characteristics. In order to achieve these, synthetic porous carbon materials with controlled porosity and pore size distribution [4] have been developed using both synthetic precursors as well as varied activation processes. These include catalytic activation of carbon precursors in presence of metals and organometallic components [5], carbonization of polymer blends with thermally unstable components [6], and carbonization of polymer aerogel [7]. However, most of these porous carbons synthesized using above mentioned methods have broad pore size distributions. Amongst all these methods, template method has been extensively applied for synthesis of microporous carbon with narrow pore size distribution and interconnected pores with various inorganic materials such as silica particles [8-10], zeolites [11, 12], alumina, minerals [13], clays $[14,15]$ as templates. The templated microporous carbons posses narrow pore size distribution and high surface areas.

Porous carbons have been synthesized in the interstitial pores of the closed-packed sub micrometer-sized silica spheres by infiltration of phenol resin and subsequent carbonization or chemical vapor deposition (CVD) of propylene gas. Knox et al. [16] used silica gel and porous glass as templates to synthesize porous carbon materials through polymerization of phenol-hexamine mixture within pores of the silica gel followed by carbonization. The resin is heat treated in nitrogen atmosphere below $1000^{\circ} \mathrm{C}$ with subsequent dissolution of the silica template producing porous carbon materials. Han, S. et al. [17] reported the synthesis of microporous carbon materials using aqueous silica sol nanoparticles as 
templates.

In the present studies, systematic efforts have been made to prepare microporous carbons of high surface area with narrow pore size distribution using synthesized silica as templates. The effect of characteristics of silica templates on the development of pore structure and pore size distribution as well as surface characteristics of prepared microporous carbon have been investigated.

\section{Experimental}

\subsection{Materials and reagents}

Commercially available novolac type, phenol-formaldehyde resin, from Gujarat phenolic, India, was used as carbon precursore. Tetraethylorthosilicate (TEOS) was used as a silica source. Hydrochloric acid ( $\mathrm{HCl})$, acetone and hydrofluoric acid HF (48\%) were of analytical grade from Qualigens fine chemicals, India.

\subsection{Preparation of Silica templates}
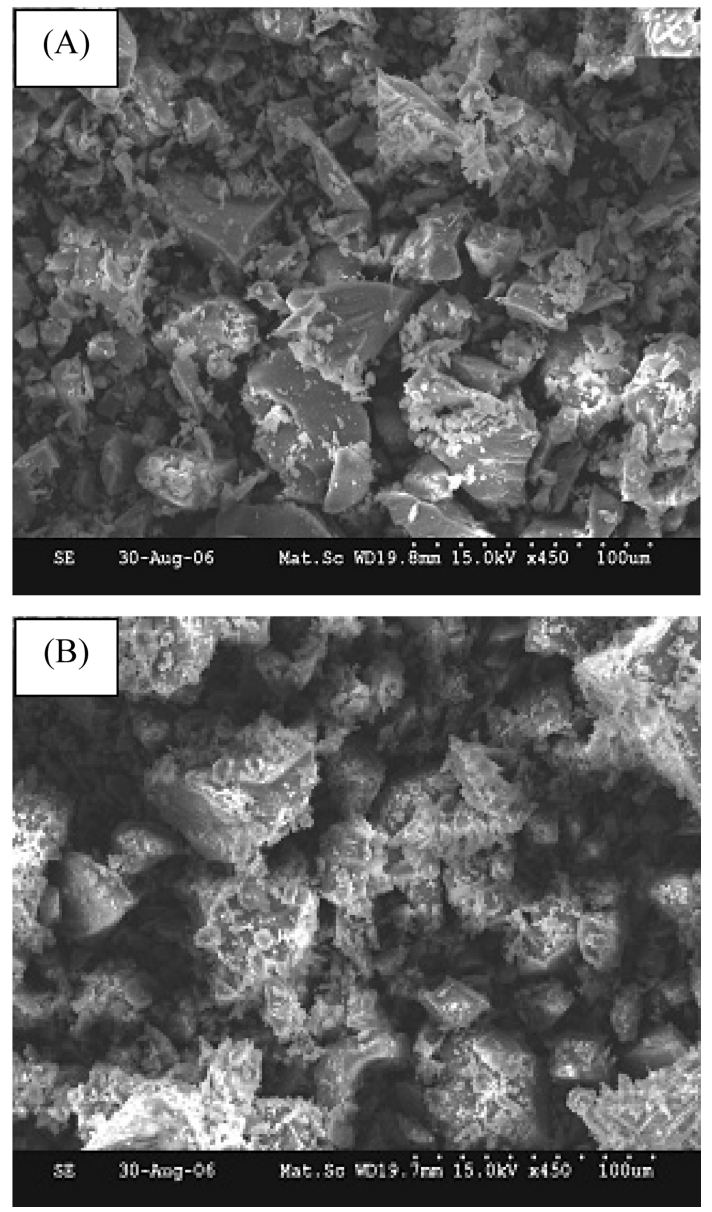

Fig. 1. SEM micrograph of template A and B.
Silica templates were prepared by hydrolysis of TEOS in presence of $\mathrm{HCl}$ as catalyst stirring it at room temperature for $12 \mathrm{~h}$. The molar ratio of TEOS, ethyl alcohol, and water was kept as 1:4:2. After completion of hydrolysis, sol was dried and were termed as template A. Fig. 1 are the SEM micrographs of the templates. The particle size distribution of template $\mathrm{A}$ is given in Fig. 2. It shows that the particle size varies between $30-80 \mu \mathrm{m}$. The dried sol powder was calcinated in air at $850^{\circ} \mathrm{C}$ for 1 hour. Its particle size distribution curve is also given in Fig. 2. The particle size varies from 25-75 $\mu \mathrm{m}$ and are termed as template B.

\subsection{Preparation of Microporous carbon}

\subsubsection{Templates A}

Template A was added to solution of phenolic resin in acetone. The mixture was homogenized and composites were prepared by pressing at $150^{\circ} \mathrm{C}$. Further process was carried out in two different ways as to have narrow pore size distribution and remove silica template as far as possible otherwise the silica adds to the formation of ash. The composites prepared with template A were processed in two ways as to remove maximum amount of silica.

(a) Phenol-formaldehyde (pf)-silica composites were carbonized at $450^{\circ} \mathrm{C}$ in nitrogen atmosphere and termed as PS1. Templates were removed from the composite by dissolution in HF. The carbon fraction was washed with distilled water to neutral $\mathrm{pH}$. Activation of carbon samples was carried out with steam at $850^{\circ} \mathrm{C}$.

(b) The second pf-silica composite designated as PS3, was carbonized at $850^{\circ} \mathrm{C}$. Templates were treated with HF. The insoluble carbon fraction was washed with distilled water to neutral $\mathrm{pH}$. Activation of silica removed carbon samples was carried out at $850^{\circ} \mathrm{C}$ with steam as an activating agent.

\subsubsection{Template B}

The composites were also prepared by using silica particles

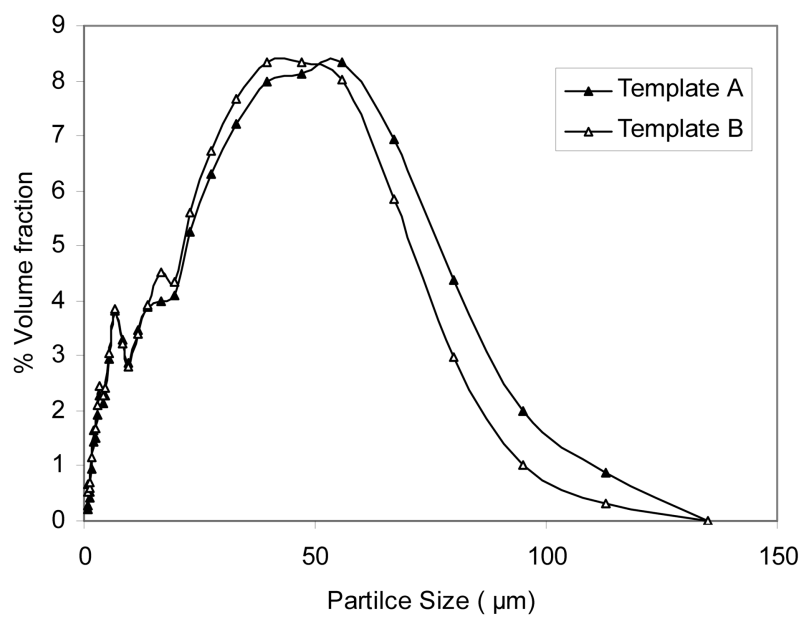

Fig. 2. Particles size distribution of template A and B. 
calcined at $850^{\circ} \mathrm{C}$ for $1 \mathrm{hr}$ in air. Two kinds of carbon samples were prepared at different carbonization temperatures.

(a) The pf-silica composites prepared, designated as PSCb1450, were carbonized at $450^{\circ} \mathrm{C}$ under nitrogen atmosphere and silica was leached out with $\mathrm{HF}$ followed by activation of carbon samples with steam at $850^{\circ} \mathrm{C}$.

(b) The second pf-silica composite, designated as PSCb1850, was carbonized at $850^{\circ} \mathrm{C}$ under nitrogen atmosphere. Silica was removed by leaching with HF. The samples were activated with steam at $850^{\circ} \mathrm{C}$.

For the comparison, porous carbons were prepared with phenol formaldehyde resin without silica template under similar preparation conditions as those with silica templates (PS1 and PS3) and are designated as P1 and P2 respectively.

\subsection{Characterization of Microporous carbon}

\subsubsection{Nitrogen adsorption isotherm}

The Micromeritics Gemini 2375 surface area analyzer was used to determine surface area and pores characteristics, e.g. micropore volume, pore size distribution and total pore volume of microporous carbon. Prior to adsorption, representative samples were degassed at $200^{\circ} \mathrm{C}$ for $4 \mathrm{hrs}$ in argon atmosphere. The micropore volume and pore size distribution were determined from adsorption branch of nitrogen isotherm using t-plot method and BJH method respectively.

\subsubsection{FTIR analysis}

The chemical structure changed by thermal treatment and silica template leaching were ascertained by Shimadzu-8300, Fourier transform infrared spectrophotometer. The spectra were recorded from 4000 to $500 \mathrm{~cm}^{-1}$ region.

\subsubsection{Iodine number}

Iodine number was studied by the adsorption of iodine from solution using $0.1 \mathrm{~N}$ standardized iodine solution. The titrant used was $0.1 \mathrm{~N}$ sodium thiosulfate [18]. Iodine number of different porous carbons developed above was measured using standard adsorption method.

\subsubsection{Thermo gravimetric analysis}

Thermo gravimetric analysis (TGA) was carried out by using Mettler TG50 system. The samples were heated in air at $20^{\circ} \mathrm{C} / \mathrm{min}$ heating rate under a flow of air from $25^{\circ} \mathrm{C}$ to $950^{\circ} \mathrm{C}$. The amount of ash formed was taken as silica present in the porous carbon.

\section{Results and Discussion}

\subsection{SEM Observation}

The SEM photographs of the two types of silica templates are shown in Fig. 1, A (Hydrolyzed silica) and B (Calcined silica). It shows that silica template has coarse grain particulate microstructure having irregular shaped polygonals. In both the templates, clear microcrystalline interfaces were observed. The calcined silica template show high sharp edge as compared to hydrolyzed silica template. The hydrolyzed silica template has hydroxyls groups on the surface. These interlink with each other and with silica tetrahedrons. They confirm that the crystallinity of calcined silica template is highest than hydrolyzed silica template. Also the particles of hydrolyzed silica template have a larger particle size as compared to calcined silica particles. The particles size was also determined by particle size analysis and are given in Fig. 2.

Figures $3 \& 4$, show the SEM photographs of the carbon samples prepared from template A and B. SEM of porous carbons shows the shape and structure, which strongly replicate both the silica templates, the most remarkable feature of the appearance of these microporous carbons is a strong resemblance to that of the corresponding templates. There seems to be almost no difference in morphology between the silica templates and their corresponding microporous carbons matrix. In the case of the porous carbon prepared with phenol formaldehyde resin only (without templates), the smooth surface of the porous carbon particles looks dense
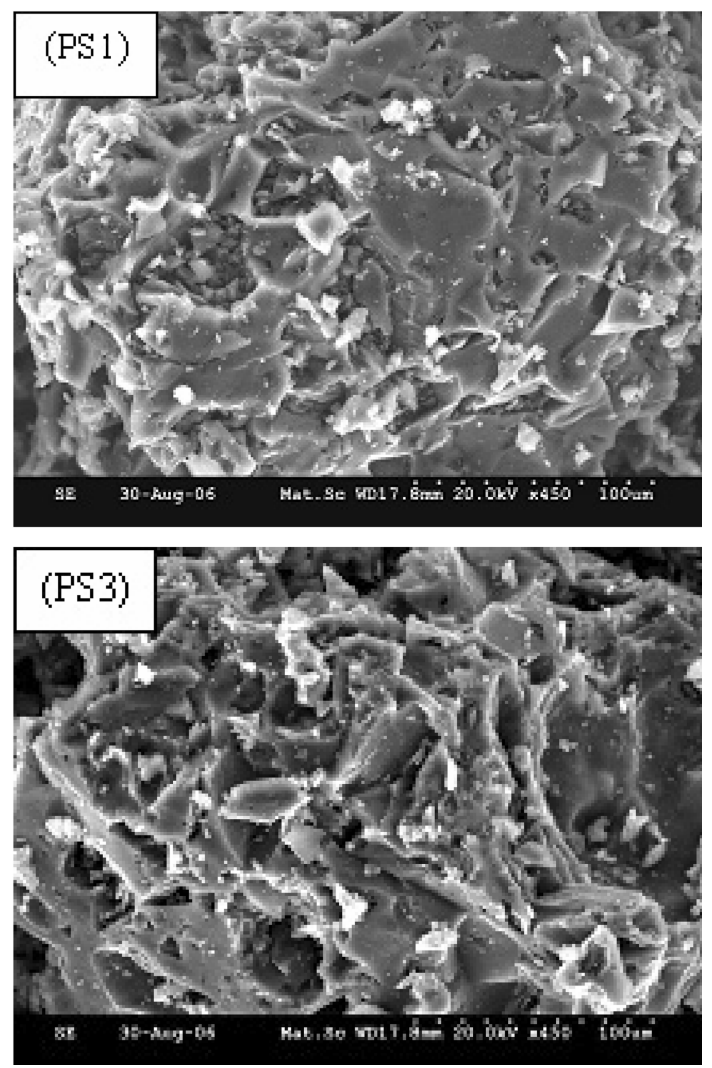

Fig. 3. SEM micrograph of microporous carbon prepared from template A. 

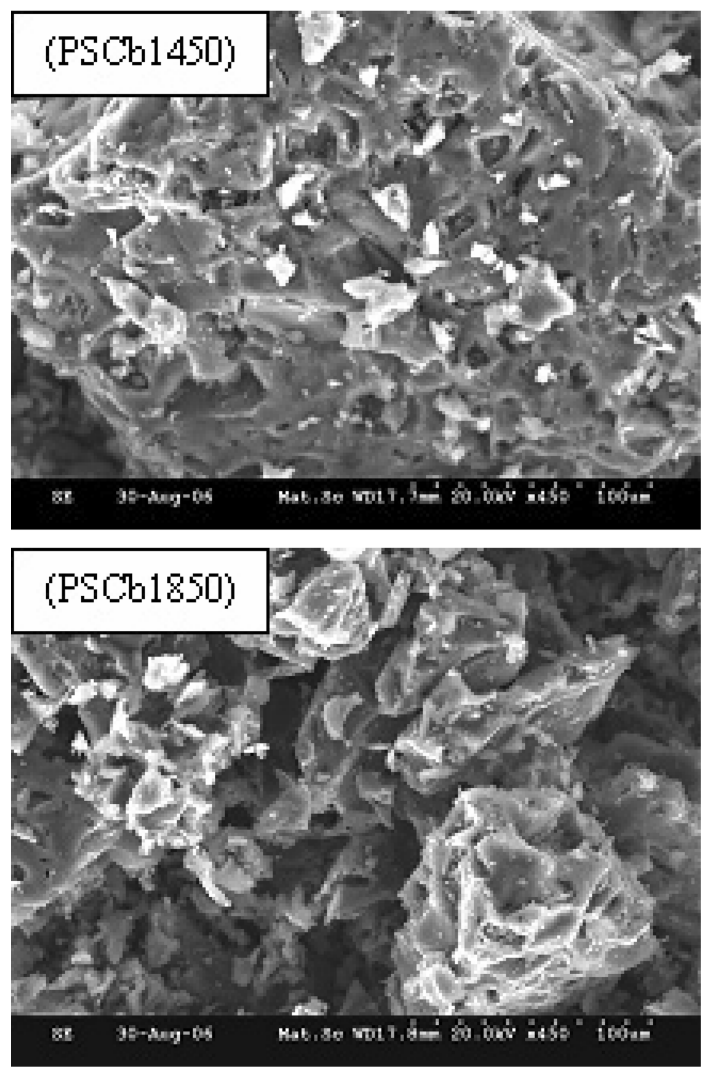

Fig. 4. SEM micrograph of microporous carbon prepared from template B.

flat and with rigid continuous phase (Fig. 5). Such a smooth and flat morphological feature was not observed in the porous carbon samples prepared with silica templates A and B. This difference in microscopic form corresponds to the morphological difference between porous carbon prepared with and without silica templates.

A comparison study shows that porous carbon prepared by using silica templates, are having $\sim 90 \%$ microporosity but pores formed with calcined silica template are sharp edged as compared to the pores formed by using hydrolyzed silica sol.

\subsection{FTIR analysis}

FTIR analysis gives results on the nature of the functional group present on the carbon samples. The surface of porous carbon, with the complex characters has been extensively studied by several workers because the chemical surface features also contributes to the specific adsorption. It is well known that activated carbons have surface oxygen groups, giving rise to the large peak between 1200 and $1000 \mathrm{~cm}^{-1}$ arising from the vibration of $\mathrm{C}-\mathrm{O}$ bonds in the carbon samples.

Typical spectra for microporous carbon prepared by using templates $\mathrm{A}$ and $\mathrm{B}$ and sample without silica templates are
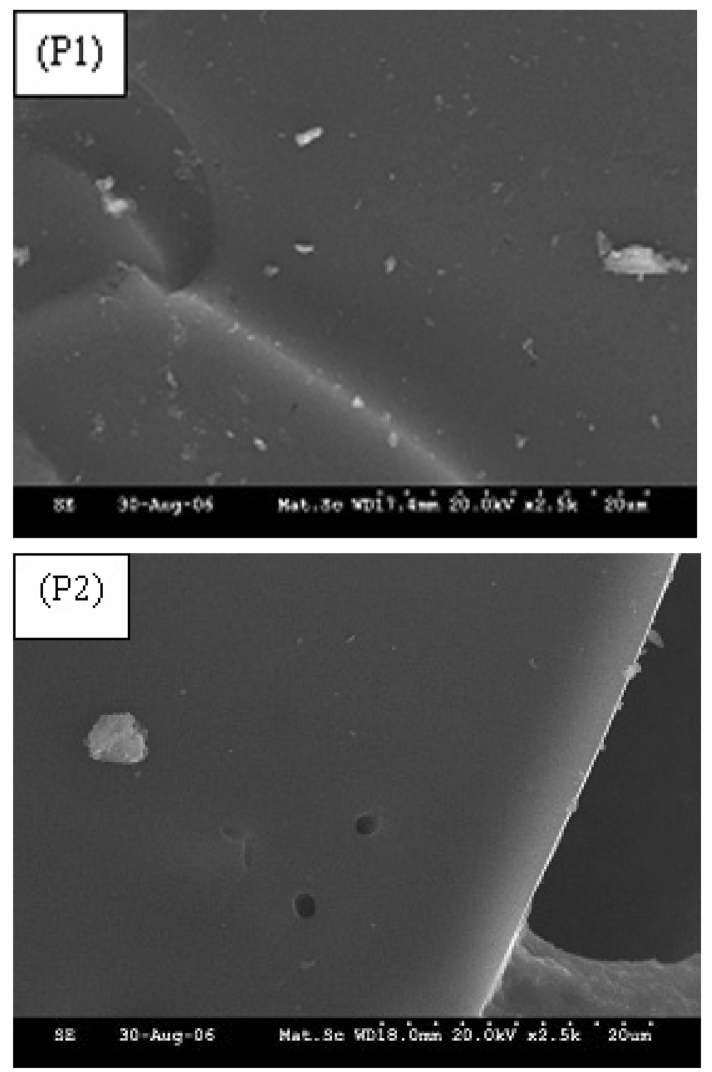

Fig. 5. SEM micrograph of porous carbon prepared without silica template.

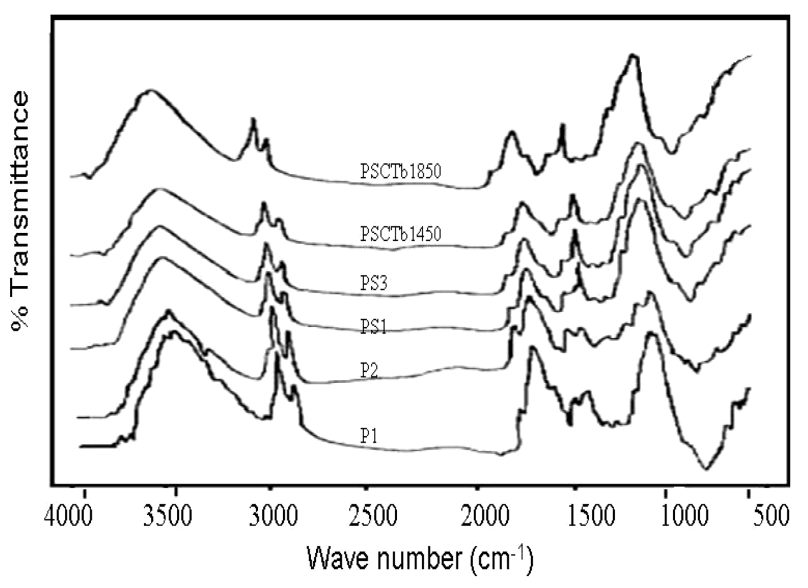

Fig. 6. FTIR spectra of the microporous carbon prepare from without template and with template A and B.

shown in Fig. 6. A significant difference between the two types of microporous carbons prepared with silica template and without silica templates is the peak at $840 \mathrm{~cm}^{-1}$. All samples prepared using templates $\mathrm{A}$ and $\mathrm{B}$ show presence of peak at $840 \mathrm{~cm}^{-1}$. This might be due to Si-O vibration in SiO-Si [21]. This peak was not seen in samples prepared without silica template. This peak is associated to the princi- 
pal band in $\mathrm{SiO}_{2}$ structure. FTIR studies show that in both carbon samples prepared with either of silica template, $\mathrm{Si}-\mathrm{O}$ components are introduced from the Si-O silica gel network. It is also observed that after removing the silica templates from carbon samples there is a band at approximately 1220 $\mathrm{cm}^{-1}$, that is perhaps a region indicative of the carbon oxygen complexes [21, 22]. These peaks are not present in carbon samples prepared without using silica templates. This peak at $1220 \mathrm{~cm}^{-1}$ can be attributed to the $\mathrm{C}-\mathrm{O}$ stretching and $-\mathrm{COOH}$ groups. It is worth noting that hetro atoms, especially oxygen, are always present on the surface of activated carbons as surface oxygen complexes [23]. The peak at $1700 \mathrm{~cm}^{-1}$ and $1720 \mathrm{~cm}^{-1}$ can be assigned to the $\mathrm{C}=\mathrm{O}$ double bonds of carboxyl groups or presence of pyrone like structures on the carbon surface. The $2920 \& 2850 \mathrm{~cm}^{-1}$ peaks are attributed to a saturated $-\mathrm{CH}_{2}$ group. The $2960 \mathrm{~cm}^{-1}$ peak is assigned to an asymmetric mode of a $-\mathrm{CH}_{3}$ group. In all recorded spectra, in the $3600-3500 \mathrm{~cm}^{-1}$ range, a peak of O-H stretch- ing vibration is observed due to the existence of surface hydroxyl groups and also may be due to the chemisorbed water [24-26]. The presence of peak characteristic of $-\mathrm{CH}_{3}$ or $-\mathrm{CH}_{2}$ structure $\left(3000-2800 \mathrm{~cm}^{-1}\right)$ in all the samples, suggest the existence of aliphatic species on the surface of carbons [24-27]. FTIR spectra of each samples indicates the presence of definite oxygen complexes containing various functional groups.

\subsection{Nitrogen adsorption and pore size analysis}

The microporous carbons were prepared using above mentioned two different silica templates viz hydrolyzed silica and calcined silica. To see the effect of the template on the formation of porosity, blank samples were also prepared. The adsorption isotherm and pore size distribution of no template porous carbon $\mathrm{P} 1$, and $\mathrm{P} 2$ are given in Fig. 7a, b. The two isotherms in Fig. 7a, and b, correspond respectively
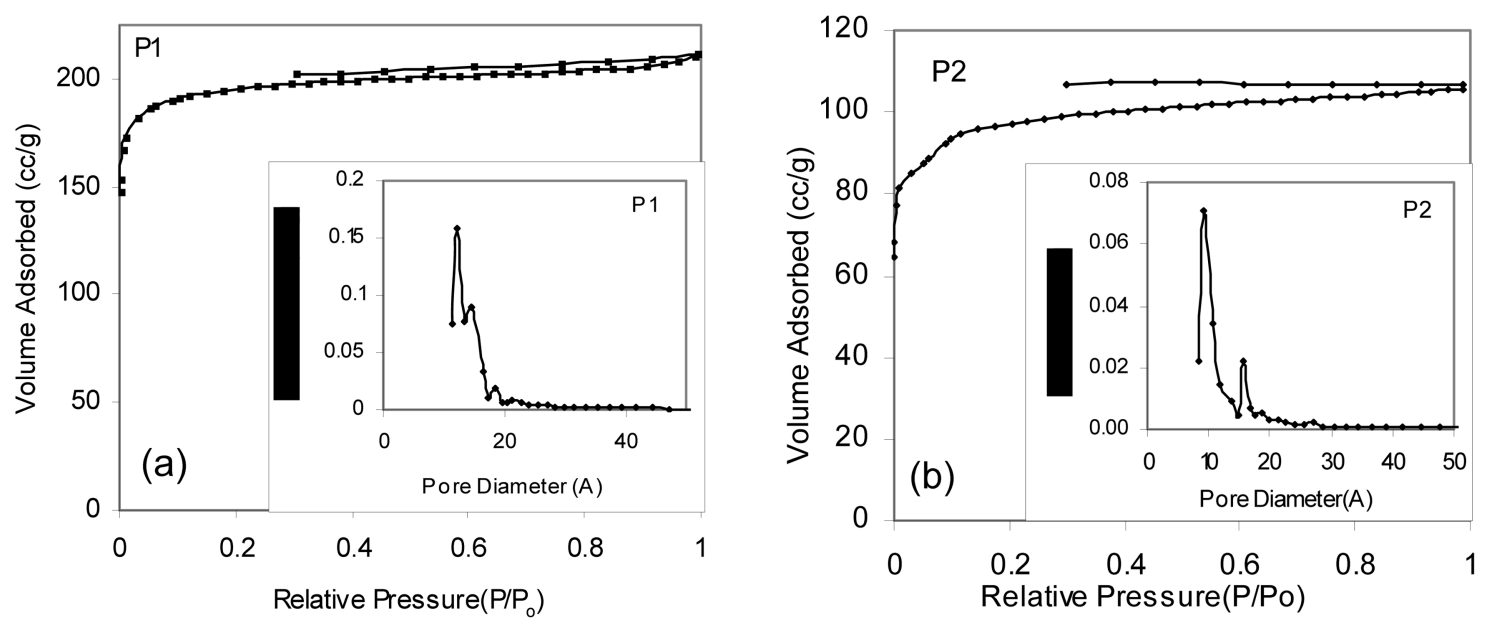

Fig. 7. Nitrogen adsorption-desorption isotherm of carbon prepared by without silica templates and inset: corresponding pore size distribution curve, (a) sample P1, (b) sample P2.
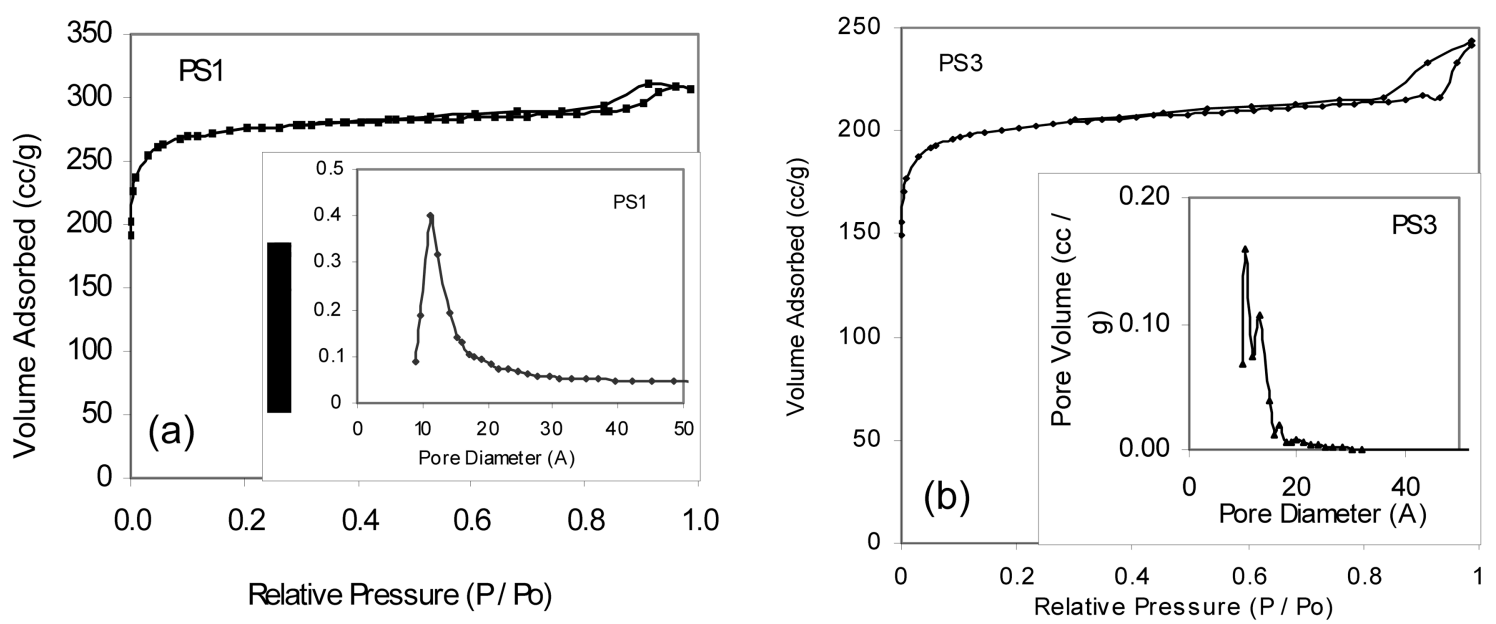

Fig. 8. Nitrogen adsorption-desorption isotherms of carbon prepared with Hydrolyzed silica particles as templates and inset: corresponding pore size distribution curve (a) sample PS1, and (b) sample PS3. 

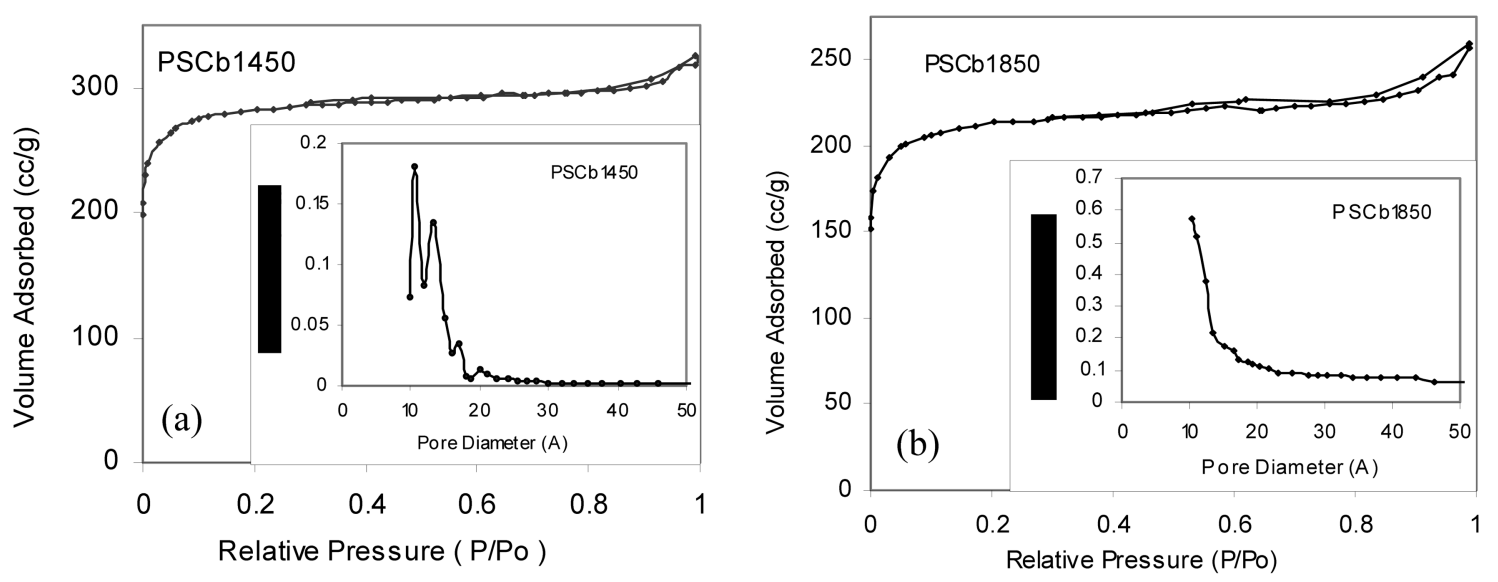

Fig. 9. Nitrogen adsorption-desorption isotherms of carbon prepared with calcined silica particles as templates and inset: corresponding pore size distribution curve (a) PSCb1450 and (b) PSCb1850.

to sample carbonized at $450^{\circ} \mathrm{C}$ and activated at $850^{\circ} \mathrm{C}$, carbonized and activated at $850^{\circ} \mathrm{C}$. All the isotherms are of type I. The steep rise in nitrogen adsorption occur at low relative pressure leading to equilibrium state indicating existence of micropores in the samples. Porous carbon prepared with template B are microporous in nature (Fig. 9), while porous carbon prepared with template A exhibited narrow hysteresis loop at high relative pressure range (Fig. 8), demonstrating the presence of some mesoporosity in the carbon samples. The hydrolyzed silica sol has hydroxyl surface groups, which forms bond with the matrix. On leaching, these weak bonds break and some of micropores become bigger and give rise to mesoporosity.

The resulting surface area and pore characteristics are given in Table 1. The BET surface area and pore volume of porous carbon samples prepared without silica templates is around $366-766 \mathrm{~m}^{2} / \mathrm{g}$ and $0.16-0.32 \mathrm{~cm}^{3} / \mathrm{g}$ respectively. By addition of template $\mathrm{A}$ and $\mathrm{B}$ to the pf resin, BET surface area and pore volume were found to increase to about 720 $1099 \mathrm{~m}^{2} / \mathrm{g}$ and $0.37-0.50 \mathrm{~cm}^{3} / \mathrm{g}$ respectively. Low surface area porous carbon was generated in the absence of silica templates. This increase in BET surface area and pore volume suggest that the template A and B play a templating role in the formation of high surface area and pore volume, especially pores in micropore range $(<2 \mathrm{~nm})$, Table 1 , shows the pore characteristic of porous carbons prepared with and without silica templates.

Pore size and pore size distribution curves of samples prepared without using template and with template $\mathrm{A}$ and $\mathrm{B}$, obtained by analyzing the adsorption branch of the nitrogen adsorption isotherm using BJH method are given in inset of Figs. 7, 8 and 9. Jaroniec and co-worker reported the broadening of these hysteresis loop mainly due to two factors: pore size distribution and pore connectivity [19, 20]. All porous carbons have pore size in micropore range, but relatively inhomogeneous pores. The surface area of these porous carbon samples varies with type of silica template i.e. hydrolyzed silica or calcined silica.

It can be seen that porous carbons (with template A \& B) pyrolyzed at low carbonization temperature i.e. $450^{\circ} \mathrm{C}$ have

Table 1. Pore characteristics of microporous carbon prepared from without template as well with templates. A and B

\begin{tabular}{|c|c|c|c|c|c|c|c|c|}
\hline Templates & Samples & $\begin{array}{c}\mathrm{S}_{\mathrm{BET}}^{\mathrm{a}} \\
\mathrm{m}^{2} / \mathrm{gm}\end{array}$ & $\begin{array}{c}\mathrm{V}^{\mathrm{b}} \\
\mathrm{cm}^{3} / \mathrm{gm}\end{array}$ & $\begin{array}{c}\mathrm{S}_{\mathrm{Mi}}^{\mathrm{c}} \\
\mathrm{m}^{2} / \mathrm{gm}\end{array}$ & $\begin{array}{c}\mathrm{V}_{\mathrm{Mi}}^{\mathrm{d}} \\
\mathrm{cm}^{3} / \mathrm{gm}\end{array}$ & $\% \mathrm{~S}_{\mathrm{Mi}}^{\mathrm{e}}$ & $\begin{array}{l}\text { Iodine Number } \\
\mathrm{mg} / \mathrm{gm}\end{array}$ & $\begin{array}{c}\text { Residue Silica }^{\mathrm{g}} \\
\%\end{array}$ \\
\hline \multirow[t]{2}{*}{ Without template } & P1 & 766 & 0.326 & 689 & 0.265 & 89.25 & 753 & - \\
\hline & P3 & 366 & 0.163 & 300 & 0.120 & 81.52 & 359 & - \\
\hline \multirow{2}{*}{$\begin{array}{l}\text { Template A } \\
\text { (hydrolyzed silica) }\end{array}$} & PS1 & 1062 & 0.474 & 953 & 0.372 & 89.81 & 1037 & 2.4 \\
\hline & PS3 & 720 & 0.374 & 626 & 0.277 & 87.00 & 728 & 3.15 \\
\hline \multirow{2}{*}{$\begin{array}{l}\text { Template B } \\
\text { (calcined silica) }\end{array}$} & PSCb 1450 & 1099 & 0.503 & 972 & 0.375 & 88.44 & 1121 & 2.12 \\
\hline & PSCb 1850 & 815 & 0.400 & 707 & 0.278 & 86.76 & 801 & 1.10 \\
\hline
\end{tabular}

${ }^{a}$ SBET is the total surface area calculated by the BET method.

${ }^{b} \mathrm{~V}$ is the total pore volume calculated by BJH method.

${ }^{\mathrm{C}} \mathrm{SMi}$ is the micropore area calculated by the t-plot method.

${ }^{\mathrm{d}} \mathrm{VMi}$ is the micro pore volume calculated by t-plot method.

${ }^{e}$ is the $\%$ microporosity presence in sample.

${ }^{\mathrm{f}} \mathrm{Measured}$ by adsorption of iodine from standardized iodine solution.

${ }^{g}$ Residue silica is silica template not removed by HF leaching. 


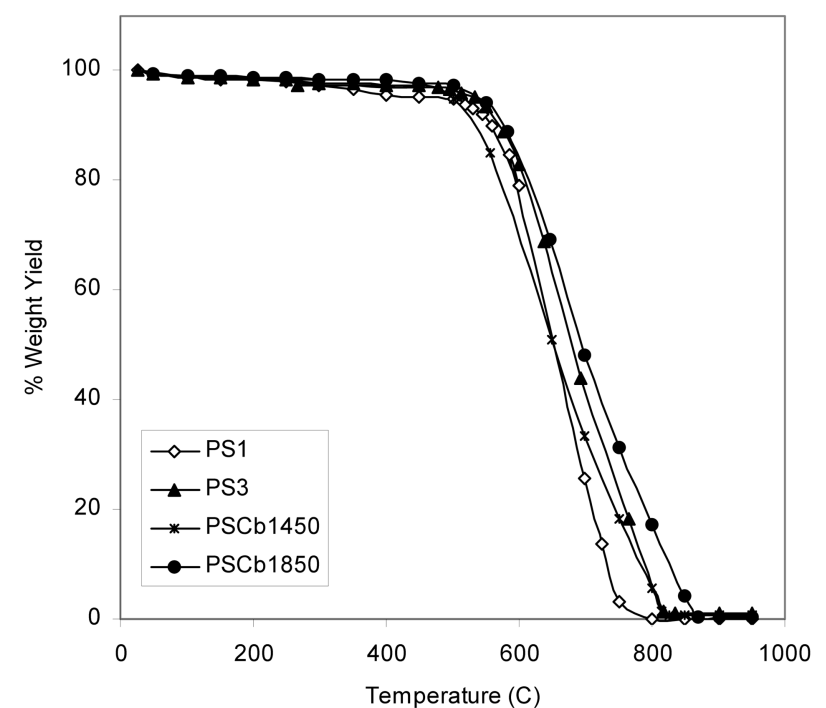

Fig. 10. TG plots of microporous carbon prepared from template $\mathrm{A}$ and $\mathrm{B}$.

higher BET surface area and pore volume than carbon pyrolyzed at high carbonization temperature i.e. $850^{\circ} \mathrm{C}$. Further, the values for micropore area and micropore volume calculated by nitrogen adsorption by t-plot method are higher in carbon prepared with template $\mathrm{B}$ carbonized at $450^{\circ} \mathrm{C}$ and activated at $850^{\circ} \mathrm{C}$. This difference in surface area and porosity show the effect of silica templates in the generation of microporosity and effectiveness in increase in surface area and pore volume.

\subsection{Iodine number}

Iodine adsorption capacity of microporous carbon prepared from template A and B are included in Table 1. The iodine adsorption capacity is also found to be nearly same and increases with increase in BET surface area. This result indicates that the volumes of smaller pores (corresponding to iodine molecule) predominate in these samples. The samples having higher Iodine number, have higher adsorption capacity and surface area.

\subsection{TG analysis}

Thermo gravimetric analysis (TGA) was carried out to ascertain the complete removal of silica, used as template from porous carbons. The results are shown in Fig. 10. The TGA results indicate that $97-99 \%$ of silica is removed by HF leaching from the samples. There was significant weight loss in a broad temperature range from $475-825^{\circ} \mathrm{C}$ in all microporous samples. The low and broad temperature range for oxidation of carbon samples indicates the non-graphitic or amorphous nature of microporous carbons. Microporous carbons prepared from phenol formaldehyde resin show low thermal stability [28].

\section{Conclusions}

The preparation of microporous carbon with narrow pore size distribution has been achieved using hydrolyzed and calcined silica templates. It has been observed that types of silica template play an important role in the pore structure development in the porous carbons. Also, the results show that calcined silica template is more effective for pore development than the template obtained from hydrolyzed silica. The presence of hydroxyl groups on surface results in formation of mesoporosity. Development of \% microporosity is found to be higher in porous carbon prepared from hydrolyzed silica than porous carbon prepared from calcined silica templates.

\section{Acknowledgement}

Authors are thankful to Department of Atomic Energy (DAE), Govt. of India for providing financial support under major research project No. 2004/37/32/BRNS/2127. Narendra Movaliya is thankful to Department of Atomic Energy (DAE), Govt. of India for providing Junior research Fellowship under the project. Authors wish to thank Sophisticated Instrumentation Centre for Advance Research and Testing (SICART), Vallabh Vidyanagar for particle size analysis.

\section{References}

[1] Patrick, J. W. "Porosity in Carbons: Characterization and Applications", Edward Arnold, London, 1995.

[2] Manocha, S.; Chauhan, V. B.; Manocha, L. M. Carbon Science 2002, 3, 133.

[3] Daud, W. M.; Badri, M.; Mansor, H. J. Appl. Phys. 1990, 67, 1915.

[4] Kyotani, T. Carbon 2000, 38, 269.

[5] Tamai, H.; Kakii, T.; Hirota, Y.; Kummamoto, T.; Yasuda, H. Chem. Mater. 1996, 8, 454.

[6] Patel, N.; Okabe, K.; Oya, A. Carbon 2002, 40, 315.

[7] Tamom, H.; Shizaka, H.; Akaki, T.; Okazaki, M. Carbon 1998, 36, 1257.

[8] Han, S.; Hyeon, T. Carbon 1999, 37, 1645.

[9] Han, S.; Hyeon, T. Chem. Commun. 1999, 1955.

[10] Han, S.; Sohn, K.; Hyeon, T. Chem. Mater. 2000, 12, 3337.

[11] Ma, Z.; Kyotani, T.; Tomita, A. Carbon 2002, 40, 2367.

[12] Su, F.; Zhao, X. S.; Lu, L.; Zhou, Z. Carbon 2004, 42, 2821.

[13] Bandosz, T. J. J.; Putyera, J. K.; Schwarz, J. A. Chem. Mater. 1996, 8, 2023. 
[14] Meyers, C. J.; Shah, S. D.; Patel, S. C.; Sneeringer, R. M. C.; Bessel, A.; Dollahon, N. R.; Leising, R. A.; Takeuchi, E. S. J. Phys. Chem. 2001, B 105, 2143.

[15] Zakidov, A.; Baughman, R.; Iqbal, Z.; Cui, C.; Khayrullin, I.; Dantas, S.; Marti, J.; Ralchenko, V. Science 1998, 282, 897.

[16] Knox, J. H.; Kaur, B.; Millward, G. R. J. Chromatogr 1986, 352, 3.

[17] Han, S.; Lee, K. T.; Oh, S. M.; Hyeon, T. Carbon 2003, $41,1049$.

[18] ASTM D 4607, 86 (Reapproved 1990).

[19] Manocha, L. M.; Yasuda, E.; Tanabe, Y.; Manocha S.; Vashista, D. Bull. Mater. Sci. 2000, 23, 1.

[20] Adib, F.; Bagreev, A.; Bandosz, T. J. Langmuir 2000, 16,
1980.

[21] Zhang, J. M.; Eklund, P. C. J. Mater. Res. 1987, 2, 858.

[23] Puri, B. R. "Chemistry and Physics of carbon", vol.6, ed. P. L. Walker. Jr. Marcel Dekker Inc. New York, 1970, 191.

[24] Biniak, S.; Szymanski, G. J.; Swiatkowski, A. Carbon 1997, 35, 1799.

[25] Hontoria-Lucas, C.; Lopaez-Peinado, A. J.; Lopez-Konzalez, J. De D; Rojas-Cervantes, M. L.; Martin Aranda, R. M. Carbon 1995, 33, 1585.

[26] Kruk, M.; Jaroniec, M.; Guan, S.; Inagaki, M. J. Phys. Chem. B 2001, 105, 681.

[27] Kruk, M.; Jaroniec, M. Chem. Mater. 2001, 13, 3169.

[28] Ryoo, R.; Joo, S. H.; Jun, S. J. Phy. Chem. B 1999, 103, 7743. 